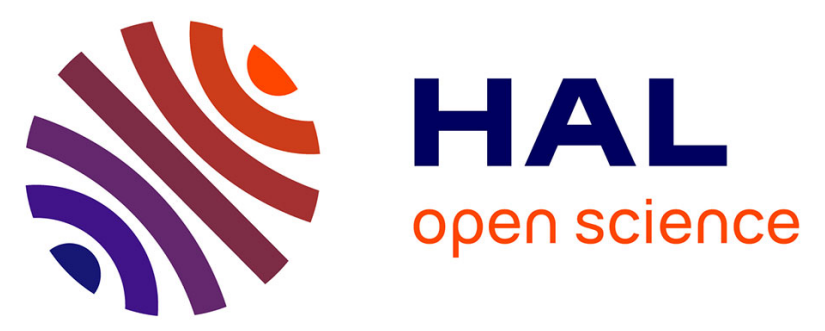

\title{
Numerical dosimetry of currents induced in the human body by ELF magnetic fields
}

\author{
Riccardo Scorretti, Ronan Perrussel, Laurent Morel, Noël Burais, Laurent
}

Nicolas

\section{- To cite this version:}

Riccardo Scorretti, Ronan Perrussel, Laurent Morel, Noël Burais, Laurent Nicolas. Numerical dosimetry of currents induced in the human body by ELF magnetic fields. COMPEL: The International Journal for Computation and Mathematics in Electrical and Electronic Engineering, 2010, 29 (6), pp.1425-1434. 10.1108/03321641011078490 . hal-00515690

\section{HAL Id: hal-00515690 \\ https://hal.science/hal-00515690}

Submitted on 4 May 2011

HAL is a multi-disciplinary open access archive for the deposit and dissemination of scientific research documents, whether they are published or not. The documents may come from teaching and research institutions in France or abroad, or from public or private research centers.
L'archive ouverte pluridisciplinaire HAL, est destinée au dépôt et à la diffusion de documents scientifiques de niveau recherche, publiés ou non, émanant des établissements d'enseignement et de recherche français ou étrangers, des laboratoires publics ou privés. 


\title{
Numerical dosimetry of currents induced in the human body by ELF magnetic fields
}

\author{
Riccardo Scorretti ${ }^{\odot}$, Ronan Perrussel ${ }^{*}$, Laurent Morel $^{\odot}$, \\ Noël Burais ${ }^{\ominus}$, Laurent Nicolas* \\ Laboratoire Ampère - UMR 5005 CNRS ; Université de Lyon, Lyon, F-69361, France. \\ ${ }^{\circ}$ Université Lyon 1, Villeurbanne, F-69622, France. \\ e-mail: Name. Surname@univ-1yon 1. fr \\ *École Centrale de Lyon, Ecully, F-69134, France. \\ e-mail: Name.Surnamedec-lyon.fr
}

April 28, 2010

\begin{abstract}
Purpose:

The classical $\phi-\mathbf{a}$ formulations for numerical dosimetry of currents induced by ELF magnetic fields requires that the source field is provided through a vector potential. We present here a new formulation $\mathbf{t}-\mathbf{b}$ which directly takes the flux density as source term.

Approach:

This formulation is implemented through Finite Element and validated by comparison with analytical solutions. The results obtained by both formulations are compared in the case of an anatomical computational phantom exposed to a vertical uniform field.

Findings:

A good agreement between the $\mathbf{t}-\mathbf{b}$ formulation and both numerical and analytical computations is found.

Originality:

This new formulation seems to be more accurate than the $\phi-\mathbf{a}$ formulation, and is more suited for situations where the magnetic field is known from experimental measurements, as there is no need for a magnetic vector potential.
\end{abstract}

Keywords: ELF, numerical dosimetry, formulation, finite element

\section{Introduction}

The fields induced in the human body by ELF electromagnetic fields are extremely weak, and hence very difficult to measure. The concerns about their possibly adverse effects, and the need to assess the respect of the maximum levels allowed by the law makes their quantification through numerical dosimetry an important issue. In numerical dosimetry, the most used formulation of magnetically induced fields is the classical $\phi-\mathbf{a}$ formulation, which takes benefit of the particular features of the human body (Dimbylow, 2000): the displacement currents and the reaction field may be neglected for low frequencies (less than $1 \mathrm{MHz}$ ). However, this formulation requires as source 
term a magnetic vector potential, and not the magnetic field itself: this can be a problem in practical situations where an accurate model of the radiating source is not available. A possible solution could be to estimate an equivalent source (Ilvonen et al., 2005), but this kind of inverse problem is likely to be ill-posed (Scorretti et al., 2005), and therefore difficult to solve. Another solution could be to solve a preliminary problem to obtain a vector potential a from the flux density $\mathbf{b}$, so as to use it as source term for the $\phi-\mathbf{a}$ formulation.

In this work, we propose a new ${ }^{1} \mathbf{t}-\mathbf{b}$ formulation for ELF numerical dosimetry which, under the same assumptions of $\phi-\mathbf{a}$ formulation, allows to compute the induced fields by taking as source term directly $\mathbf{b}$. The counterpart is that the computational cost is higher than for the $\phi-\mathbf{a}$ formulation, in that the unknown is a vector potential (instead of a scalar potential). We stress that in both formulations the resolution domain is bounded to the human body - conversely to general purpose formulations (Renhart et al., 1988) like $\mathbf{t}-\omega$ or $\mathbf{a}-v$.

\section{Formulations}

\subsection{The $\phi-$ a formulation}

Let a source magnetic vector potential $\mathbf{a}$ such that $\mathbf{b}=\operatorname{curl} \mathbf{a}$. It is assumed that $\mathbf{b}$ is not modified by the presence of the human body (namely $\Omega$ ), therefore $\mathbf{a}$ is known $a$ priori. From curl $\mathbf{e}=-\partial_{t} \mathbf{b}$ one obtains:

$$
\mathbf{e}=-\partial_{t} \mathbf{a}-\operatorname{grad} \phi
$$

and

$$
\mathbf{j}=\sigma \mathbf{e}
$$

The scalar potential $\phi \in \mathrm{H}(\operatorname{grad}, \Omega)$ is the unknown. Displacement currents are neglected; from curl $\mathbf{h}=\mathbf{j}$ one obtains the charge conservation equation:

$$
\operatorname{div} \mathbf{j}=0
$$

This latter equation is imposed weakly. Green's formula for grad-div leads to a a weak form of the formulation (notation: $\left.(f, g)=\int_{\Omega} f(\mathbf{x}) g(\mathbf{x}) d \mathbf{x}\right)$ :

$$
(\sigma \operatorname{grad} \phi, \operatorname{grad} w)+\left(\sigma \partial_{t} \mathbf{a}, \operatorname{grad} w\right)=0 \forall w \in \mathrm{H}(\operatorname{grad}, \Omega) .
$$

In this equation, the surface term $\int_{\partial \Omega} \mathbf{n} \cdot \mathbf{j} w \mathbf{d x}=0$ due to the boundary condition $\mathbf{n} \cdot \mathbf{j}=0$. At the discrete level $\phi$ is approximated by nodal functions, hence a gauge is simply fixed by imposing the value of $\phi$ in a node.

\subsection{The $\mathrm{t}-\mathrm{b}$ formulation}

Let a source magnetic flux density $\mathbf{b}$, and assume that it is not modified by the presence of the human body. From curl $\mathbf{h}=\mathbf{j}$ one obtains $\operatorname{div} \mathbf{j}=0$ and therefore:

$$
\mathbf{j}=\operatorname{curl} \mathbf{t}
$$

\footnotetext{
${ }^{1}$ A somehow similar formulation can be found in a paper by Sekino and Ueno (Sekino and Ueno, 2004), but it is not for induced currents.
} 


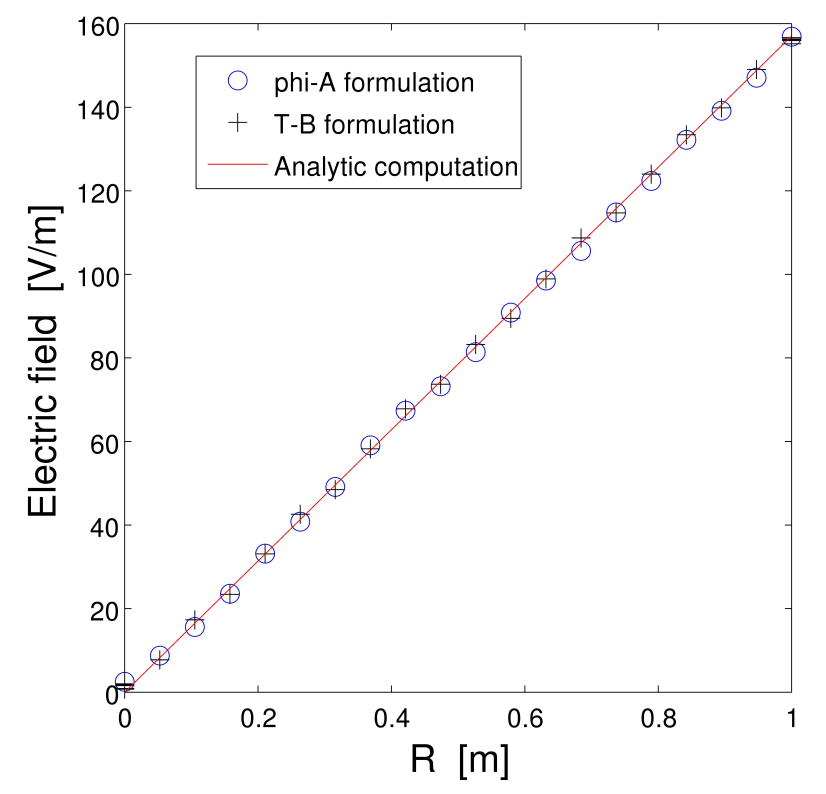

Figure 1: Induced current in a homogeneous disk computed by the $\phi-\mathbf{a}$ formulation $(\circ), \mathbf{t}-\mathbf{b}$ formulation $(+)$ compared to the analytical computation (straight line).

The vector potential $\mathbf{t} \in \mathrm{H}(\mathrm{curl}, \Omega)$ is the unknown. The equation curl $\mathbf{e}=-\partial_{t} \mathbf{b}$ can be written as:

$$
\operatorname{curl} \frac{1}{\sigma} \operatorname{curl} \mathbf{t}=-\partial_{t} \mathbf{b}
$$

This latter equation is imposed weakly. Green's formula for curl-curl leads to a weak form of the formulation:

$$
\left(\frac{1}{\sigma} \operatorname{curl} \mathbf{t}, \operatorname{curl} \mathbf{w}\right)+\left(\partial_{t} \mathbf{b}, \mathbf{w}\right)=0 \forall \mathbf{w} \in \mathrm{H}_{0}(\operatorname{curl}, \Omega)
$$

The condition $\mathbf{n} \cdot \mathbf{j}=0$ implies that $\mathbf{n} \times \mathbf{t}=0$ on the boundary of $\Omega$.

At the discrete level $\mathbf{t}$ is approximated by edge functions: therefore all the degrees of freedom on $\partial \Omega$ are set to 0 . Anyway, this is not enough to ensure the unicity of $\mathbf{t}$, and hence the stability of the numerical scheme. This can be achieved by imposing $\int_{e} \mathbf{t} \mathbf{d x}$ on all edges $e \in \mathcal{T}$ where $\mathcal{T}$ is a complete ${ }^{2}$ tree spanning over all the nodes of the mesh on $\partial \Omega$ (Dular, 1996). Observe that this is not the only way to impose a gauge to $\mathbf{t}$.

\section{Validation with analytical solutions}

Both $\phi-\mathbf{a}$ and $\mathbf{t}-\mathbf{b}$ formulations are implemented by using MATLAB and the GETFEM++ library (Renard and Pommier, 2009). Comparisons with simple (exact) analytical solutions have been performed to validate the formulations.

\footnotetext{
${ }^{2} \mathrm{~A}$ tree $\mathcal{T}$ is said to be complete on a surface $\Gamma$, when the subset of $\mathcal{T}$ belonging to $\Gamma$ is a tree spanning over all the nodes of $\Gamma$.
} 


\subsection{Homogeneous disk}

A preliminary validation has been done by using a homogeneous disk exposed to a uniform orthogonal flux density. For this simple configuration, an analytical solution exists for the induced current density:

$$
\|\mathbf{j}\|=\pi \sigma f\|\mathbf{b}\| r
$$

where $f$ is the frequency, $\sigma$ is the conductivity and $r$ is the distance from the lengths of the axis of the disk. We have computed this configuration by using both formulations, and also with the software FLUX3D $(\mathbf{t}-\omega$ formulation). The plots of $\|\mathbf{j}\|$ as a function of $r$ obtained by these formulations (figure 1) are practically superposed to the analytical solution.

6939 elements

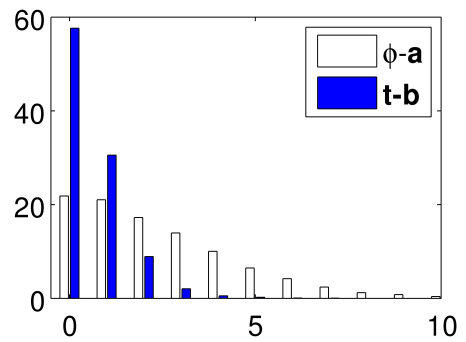

130482 elements

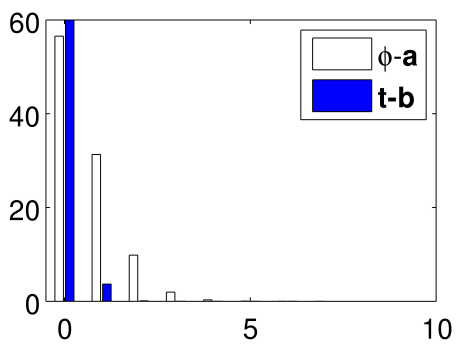

40661 elements

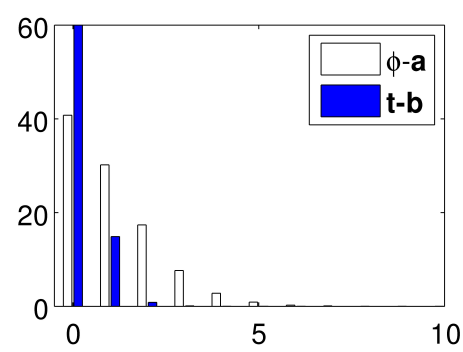

324243 elements

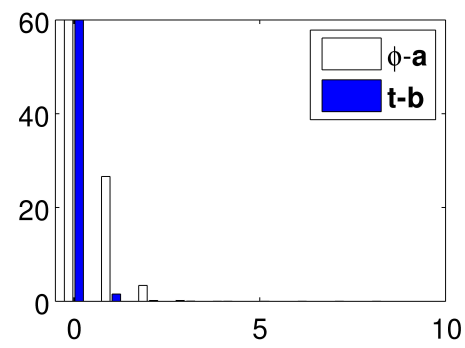

Figure 2: Error distribution on the induced current obtained with the $\phi-\mathbf{a}$ (white) and $\mathbf{t}-\mathbf{b}$ (blue) formulations, for different meshes of the sphere. The values on $\mathbf{a b}-$ scissa (namely $x$ ) represent the percent error with respect of the analytical solution; the height of bars represents the fraction of elements (also in percent) for which the error is smaller than $x \%$.

\subsection{Homogeneous sphere}

A second test has been performed with an homogeneous sphere exposed to a $50 \mathrm{~Hz}$ vertical flux density of $1 \mathrm{~T}$, for which a simple exact analytical formula for $\mathbf{j}_{\mathbf{e}}$ exists:

$$
\left\|\mathbf{j}_{\mathbf{e}}\right\|=\pi \sigma f\|\mathbf{b}\| \sqrt{x^{2}+y^{2}}
$$

So as to estimate the quality of the solution, we computed $\|\mathbf{j}\|$ in the center of each element for each formulation (Ducreux et al., 2009), and we computed it with the analytical formula (9). The considered sphere has a conductivity $\sigma=0.2 \mathrm{~S} \cdot \mathrm{m}^{-1}$. 
Table 1: Errors obtained with several meshes of the sphere

\begin{tabular}{llllll}
\hline \hline Nb. of elements & & 6939 & 40661 & 130482 & 324243 \\
\hline Mean & $\phi-\mathbf{a}$ & $3.7 \%$ & $2.0 \%$ & $1.3 \%$ & $1.0 \%$ \\
& $\mathbf{t}-\mathbf{b}$ & $1.4 \%$ & $0.8 \%$ & $0.5 \%$ & $0.4 \%$ \\
\hline Max & $\phi-\mathbf{a}$ & $22.9 \%$ & $13.0 \%$ & $10.0 \%$ & $6.2 \%$ \\
& $\mathbf{t}-\mathbf{b}$ & $54.0 \%$ & $8.1 \%$ & $8.5 \%$ & $22.9 \%$ \\
\hline 99 perc. & $\phi-\mathbf{a}$ & $12.7 \%$ & $6.7 \%$ & $4.4 \%$ & $3.1 \%$ \\
& $\mathbf{t}-\mathbf{b}$ & $5.0 \%$ & $2.6 \%$ & $1.7 \%$ & $1.5 \%$ \\
\hline \hline
\end{tabular}

The computation has been performed with different meshes for both $\phi-\mathbf{a}$ and $\mathbf{t}-\mathbf{b}$ formulations, so as to study the error distribution and the convergence of the numerical methods. The error with respect to the exact solution $\mathbf{j}_{\mathbf{e}}$ has been computed as:

$$
e=\frac{\|\mathbf{j}\|-\left\|\mathbf{j}_{e}\right\|}{\max \left\|\mathbf{j}_{e}\right\|} \times 100 \%
$$

The obtained results have been summarized in the table 1 and figure 2. Both formulations seem to converge towards the true solution as the number of elements increases, and that the order of convergence is similar for both formulations (i.e. the error decrease following a power law with the same exponent). It can be observed than the mean and 99 percentile error is better for $\mathbf{t}-\mathbf{b}$ formulation than for $\phi-\mathbf{a}$ formulation. Conversely, the maximum error has a more erratic behavior. This fact is visually confirmed by the plot of error distributions (figure 2), where it can be observed that (for the same mesh) for most of elements the error obtained by the $\mathbf{t}-\mathbf{b}$ formulation is smaller than the one obtained by the $\phi-\mathbf{a}$ formulation. Perheaps this increased accuracy of $\mathbf{t}-\mathbf{b}$ formulation may be explained by the fact that this formulation imposes strongly the continuity of $\mathbf{n} \cdot \mathbf{j}$ across regions with different conductivities. Conversely the $\phi-\mathbf{a}$ formulation imposes strongly $\mathbf{n} \cdot \mathbf{b}$, which in this particular context is "for free" in that the human body has a negligible influence on the source magnetic field.

\section{Computation with an anatomical phantom}

\subsection{Exposure to a uniform vertical field}

As a first benchmark with a realistic geometry, we performed a first computation at $50 \mathrm{~Hz}$ by using an anatomical phantom irradiated by a $1 \mathrm{mT}$ uniform vertical magnetic field. The computational phantom named "ZOL" is derived from the Visible Human project (Ackerman, 1998), and it is described in more details in (Hoang et al., 2009). The conductivities have been taken from the classical works by Gabriel et al. (Gabriel et al., 1996). In this case, the same computation has not been performed with FLUX3D due to difficulties for importing the mesh of the phantom. The surface current density obtained with the two formulations is plotted in figure 3 . The number of unknowns is 84293 for the $\phi-\mathbf{a}$ formulation, and 567299 for the $\mathbf{t}-\mathbf{b}$ formulation.

The results obtained for the average and maximum induced current $\|\mathbf{j}\|$ with both formulations are compared in table 2. For most of large organs, the average values are quite similar. Conversely, for smaller organs, the obtained values are very different. Notably, in the case of eyes the difference between the two formulations is of the order 


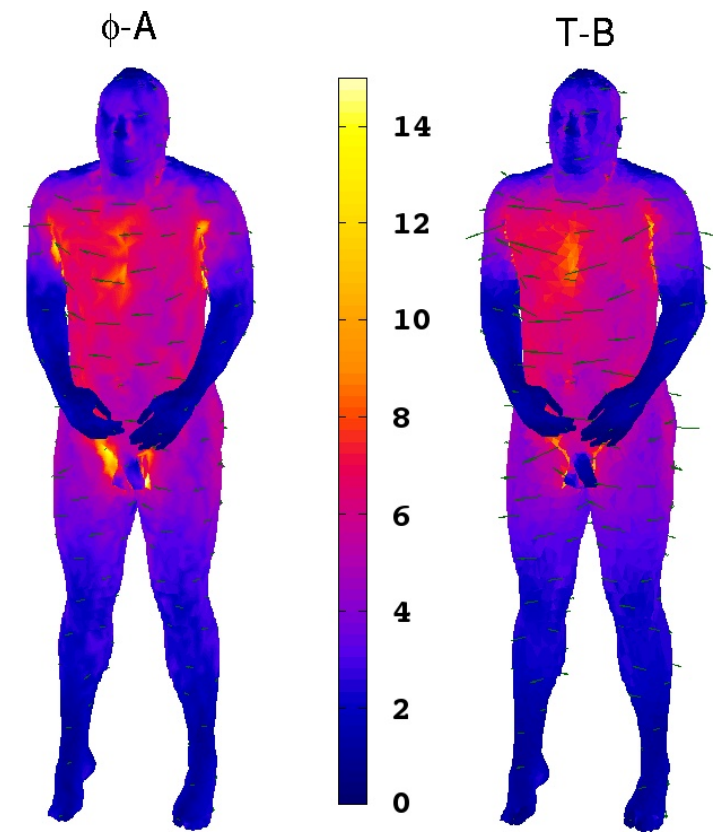

Figure 3: Induced current (in $\mathrm{mA} / \mathrm{m}^{2}$ ) in the body by a uniform vertical field ( $1 \mathrm{mT}$, $50 \mathrm{~Hz}$ ) by using the $\phi-\mathbf{a}$ (left) and $\mathbf{t}-\mathbf{b}$ formulation (right).

of $300 \%$. The same trend is observed (amplified) for maximum values. Some differences among the formulations are observed at the surface of the pelvis. At present time we have no other explication, but the presence of some badly shaped elements. Indeed, it has been observed with simple benchmarks that the $\phi-\mathbf{a}$ formulation is more sensitive to the presence of slivers than other formulations (Ducreux et al., 2009).

\subsection{Exposure to a HTA/BT transformer}

So as to provide a more realistic situation, we considered the case of a worker exposed to a $2.5 \mathrm{MW}$ three-phase power transformer. The transformer is an HTA/BT model $(20 \mathrm{kV}-400 \mathrm{~V}, \triangle-\mathrm{Y})$ at no load condition. As such a device was not practically available, the magnetic field generated in the air has been computed by using FLUX3D. Only the HV side of the device (coils and power line) has been modeled. The dimensions of the kernel have been chosen based upon data sheets of similar commercial devices, as well as the currents in the coils and in the power line (no load current $I_{0}=0.7 \mathrm{~A}$ ). Eddy current losses in the magnetic kernel have not been taken into account explicitly, so that the computation of the flux density has been done by using the standard (total and reduced) magnetic scalar potential formulations; these formulations allow to compute the flux density but not a magnetic vector potential, so that the $\phi-\mathbf{a}$ formulation cannot be used directly.

The human body is supposed to be back to the transformer under the power line on the HV side, in the exposure area represented in figure 4. The flux density in this area has been exported in a separate file, and interpolated on the nodes of the computational phantom so as to be used as source term for the $\mathbf{t}-\mathbf{b}$ formulation. The induced current at the surface of the body is represented in figure 5 . 
Table 2: Average and max current density induced in the different organs by a uniform field of $1 \mathrm{mT}$ at $50 \mathrm{~Hz}\left(\mathrm{~mA} / \mathrm{m}^{2}\right)$

\begin{tabular}{lllll}
\hline \hline & Aver. $\|\mathbf{j}\|$ & \multicolumn{3}{c}{ Max $\|\mathbf{j}\|$} \\
& $\phi-\mathbf{a}$ & $\mathbf{t}-\mathbf{b}$ & $\phi-\mathbf{a}$ & $\mathbf{t}-\mathbf{b}$ \\
\hline Muscle & 2.84 & 2.76 & 17.40 & 15.80 \\
Brain & 0.38 & 0.41 & 0.84 & 2.40 \\
Cerebellum & 0.45 & 0.46 & 0.82 & 1.21 \\
Eyes & 9.57 & 3.58 & 15.04 & 5.76 \\
Spinal marrow & 0.24 & 0.44 & 0.55 & 1.48 \\
Lungs & 0.79 & 0.85 & 2.02 & 2.79 \\
Heart & 1.00 & 1.29 & 2.51 & 6.74 \\
Veins & 4.37 & 3.11 & 20.20 & 16.58 \\
Liver & 0.54 & 0.59 & 1.41 & 2.50 \\
Stomach & 4.32 & 3.63 & 15.73 & 8.82 \\
Kidneys & 1.12 & 1.28 & 2.00 & 2.66 \\
Spleen & 1.21 & 1.36 & 1.64 & 2.42 \\
Bile & 9.12 & 3.17 & 30.62 & 8.15 \\
Colon & 0.98 & 1.13 & 2.00 & 3.47 \\
Intestine & 5.83 & 4.61 & 17.41 & 17.99 \\
Duodenum & 2.80 & 2.26 & 6.11 & 4.84 \\
Bones & 0.50 & 0.66 & 1.93 & 3.34 \\
\hline \hline
\end{tabular}

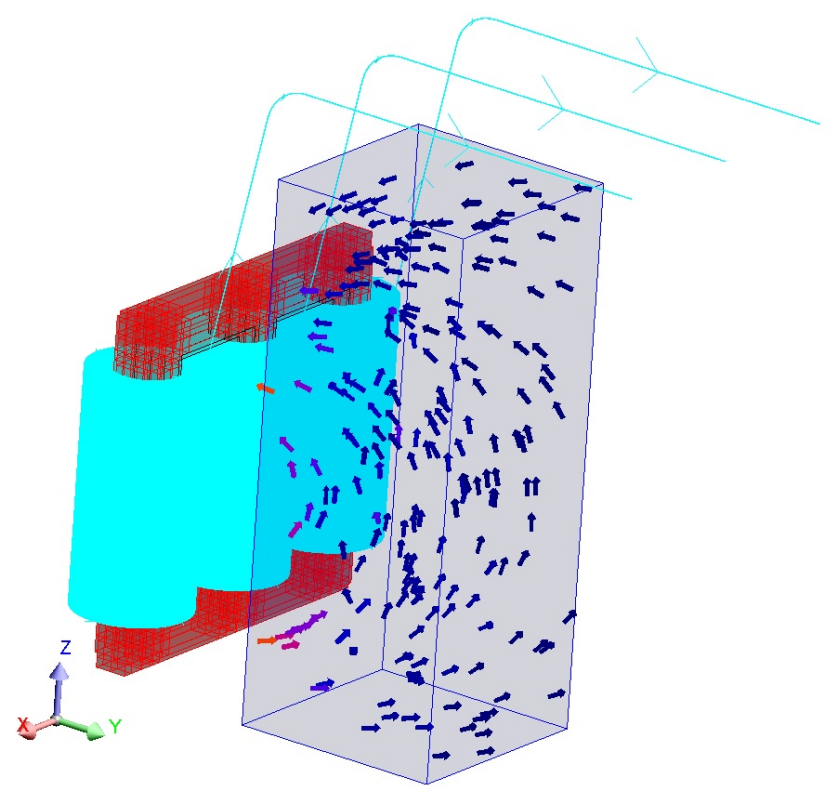

Figure 4: Model of the HTA/BT transformer (red: kernel, turquoise: primary coils and power lines) and flux density $\mathbf{b}$ (blue arrows) in the exposure area (the darker box) computed by FLUX3D. 


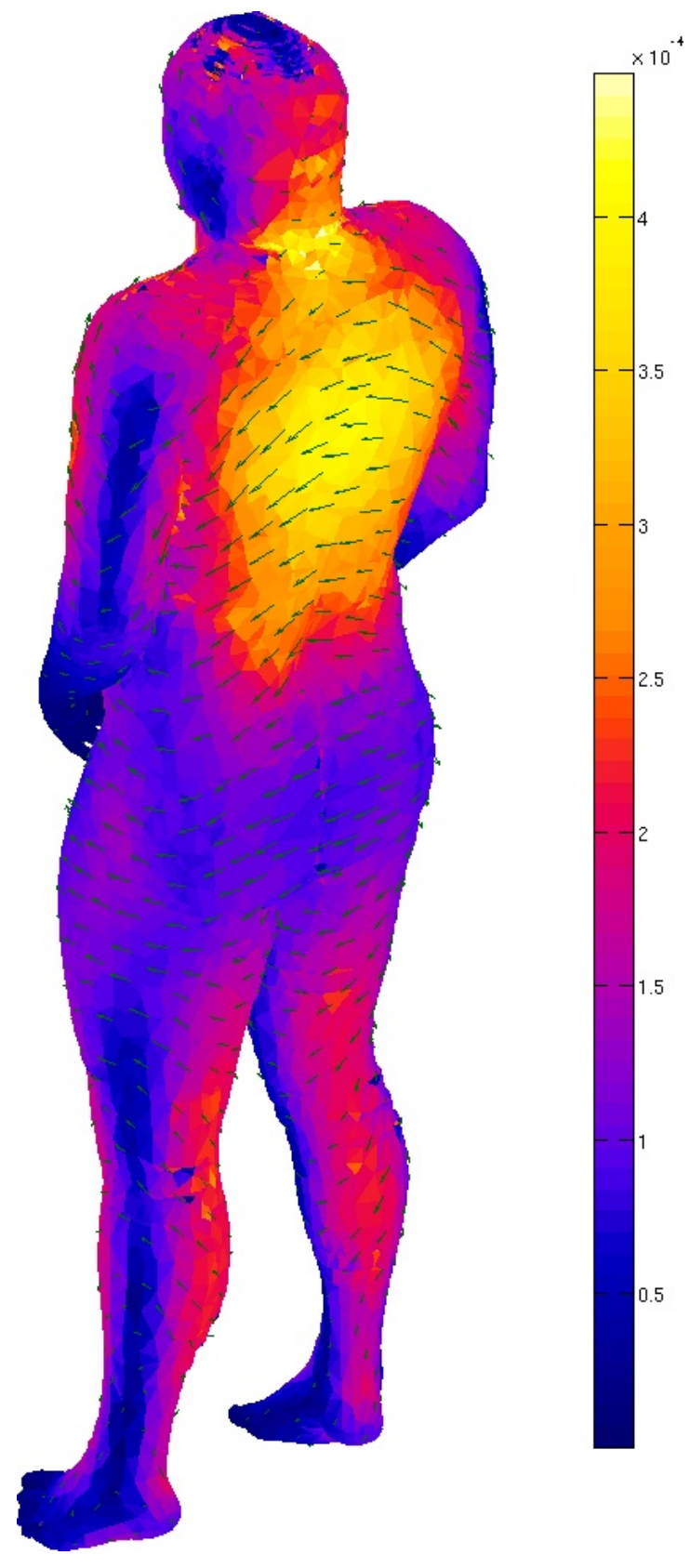

Figure 5: Induced current at the surface of the human body computed by the $\mathbf{t}-\mathbf{b}$ formulation (unit $=\mathrm{mA} / \mathrm{m}^{2}$ ). 


\section{Conclusion}

The new $\mathbf{t}-\mathbf{b}$ formulation can be effectively used for numerical dosimetry of ELF electromagnetic field in the human body. Its computational cost is higher than the classical $\phi-\mathbf{a}$ formulation. On the other hand, our results suggest that for a same mesh the $\mathbf{t}-\mathbf{b}$ formulation is more accurate than the $\phi-\mathbf{a}$ formulation. Moreover, it can be used also in cases where a (source) magnetic vector potential is either unavailable, or very difficult to obtain.

When applied to an anatomical computational phantom, some large differences appear between the results obtained with both formulations in small organs. At present time, no firm conclusion can be drawn on which formulation is more accurate (even if some clues indicate that $\mathbf{t}-\mathbf{b}$ should be preferred).

Most of the computational time has been spent for the inversion of the linear system: at present time, this topic has not been deeply studied. Therefore the computational time is likely to decrease by using more adapted solvers (which take into account the features of the curl-curl operator). Nonetheless, no efficient solver seems straightforwardly usable for the kind of gauge we choose (tree-cotree, see subsection 2.2); consequently other gauging techniques will also be considered. To this aim, another point which merits to be investigated is the influcence on the convergence rate of the right-hand side of the linear system (namely the term $\left.\left(\partial_{t} \mathbf{b}, \mathbf{w}\right)\right)$ which has to be divergency free. It has been shown that if this condition is not met, for instance due to numerical errors, poor convergence may be experienced (Ren, 1996).

\section{Acknowledgment}

The authors would like to thank Patrick Dular and Yves Renard for many fruitful discussions.

\section{References}

Ackerman, M. (1998), 'The visible human project', Proceedings of the IEEE 86(3), 504-511.

Dimbylow, P. (2000), 'Current densities in a $2 \mathrm{~mm}$ resolution anatomically realistic model of the body induced by low frequency electric fields', Physics in medicine and biology 45, 1013-1022.

Ducreux, J., Guillot, Y., Thomas, P., Burais, N. and Scorretti, R. (2009), 'Benchmarks for computing induced currents in the human body by ELF electric and magnetic fields'.

URL: http://hal.archives-ouvertes.fr/hal-00414270/fr/

Dular, P. (1996), Modélisation du champ magnétique et des courants induits dans des systèmes tridimensionnels non linéaires, $\mathrm{PhD}$ thesis, Université de Liège.

Gabriel, S., Lau, R. and Gabriel, C. (1996), 'The dielectric properties of biological tissues: III. Parametric models for the dielectric spectrum of tissues', Physics in Medicine and Biology 41, 2271-2293. 
Hoang, L., Scorretti, R., Burais, N. and Voyer, D. (2009), 'Numerical Dosimetry of Induced Phenomena in the Human Body by a Three-Phase Power Line', IEEE Transactions on Magnetics 45(3), 1666-1669.

Ilvonen, S., Sihvonen, A., Kärkkäinen, K. and Sarvas, J. (2005), 'Numerical assessment of induced ELF currents in the human head due to the battery current of a digital mobile phone', Bioelectromagnetics 26(8), 648-656.

Ren, Z. (1996), 'Influence of the RHS on the convergence behaviour of the curl-curl equation', IEEE Transactions on Magnetics 32(3 Part 1), 655-658.

Renard, Y. and Pommier, J. (2009), Getfem++: a finite element library.

URL: http://home.gna.org/getfem

Renhart, W., Stogner, H. and Preis, K. (1988), 'Calculation of 3 D eddy current problems by finite element method using either an electric or a magnetic vector potential', IEEE Transactions on Magnetics 24(1), 122-125.

Scorretti, R., Burais, N., Nicolas, L. and Nicolas, A. (2005), 'Modeling of induced current into the human body by low-frequency magnetic field from experimental data', IEEE Transactions on Magnetics 41(5), 1992-1995.

Sekino, M. and Ueno, S. (2004), 'FEM-based determination of optimum current distribution in transcranial magnetic stimulation as an alternative to electroconvulsive therapy', IEEE Transactions on Magnetics 40(4 Part 2), 2167-2169. 